\title{
Miastenia Gravis (M.G.)
}

\author{
Orlando Restrepo Ochoa*
}

\begin{abstract}
RESUMEN: Se hace una revisión que comprende la epidemiología, inmunopatología, clasificación, manifestaciones clínicas, diagnóstico y diferentes modalidades terapéuticas de la Miastenia Gravis (M.G.).

Igualmente se revisan los efectos de la M.G. sobre el embarazo y el efecto del embarazo sobre la M.G., haciendo hincapié en el manejo de esta entidad durante el embarazo, trabajo de parto y puerperio.
\end{abstract}

PALABRAS CLAVES: Acetilcolina, colinesterasa, neostigmina, piridostigmina, prednisona, timectomía, plasmaféresis.

SUMMARY: I have done a review with the following topics: epidemiology, inmunopatology, clasification, clinical approach, diagnosis and different therapies of the Myasthenia Gravis.

At the some time I review the effects of Myasthenia Gravis on the pregnant women and the effect of pregnancy on Myasthenia Gravis. I make special mention on the treatment of the Myasthenia Gravis during pregnancy, labor and puerperium.

KEY WORDS: Acetylcholine, cholinesterase, neostigmine, piridostigmine, prednisone, thymectomy, plasmapheresis.

\section{Definición}

La M.G. es una enfermedad autoinmune con predisposición genética, que clínicamente se manifiesta por debilidad y fácil fatigabilidad de grupos musculares esqueléticos, principalmente los de la cara y las extremidades, con tendencia a la recuperación tras un período de reposo o con la administración de fármacos anticolinesterasa (A-C). Aunque la primera descripción de la enfermedad se hizo en 1672 por Thomas Willis, su relación con una patología del timo sólo se estableció en 1901 (1).

\section{Epidemiología}

La enfermedad se presenta en todas las razas y su prevalencia se estima entre 2 y $10 / 100.000$; ocurre en todas las edades con mayor incidencia entre los 30 y los 40 años. La relación entre la mujer y el hombre es de dos a uno, pero en etapas tempranas de la vida es de cuatro a uno, igualándose en la senilidad.

Engle y col. suponen que la M.G. puede ser la manifestación clínica de tres enfermedades diferentes de tipo genético:

Una forma infantil autosómica recesiva, una forma hereditaria ligada al cromosoma X (que ocurre en mujeres no blancas durante la pubertad) y una forma adquirida (que padecen los hombres añosos de todas las razas) (2).

Los estudios de histocompatibilidad (HLA) y de gemelos sugieren la existencia de factores genéticos en la M.G.; es así como se ha demostrado una frecuente asociación entre la enfermedad y los haplotipos A, Be y DW del sistema de HLA. Igualmente se han descrito vínculos genéticos entre la M.G. y elementos del sistema de antígenos leucocíticos humanos (1-2).

Profesor. Departamento de Obstetricia y Ginecología. Facultad de Medicina. Universidad de Antioquia.

\section{Inmunopatología}

El mecanismo patogénico se caracteriza por la destrucción de los receptores de acetilcolina (Aco) de la membrana postsináptica de la placa motora por parte de anticuerpos fijadores de complemento; en consecuencia, la membrana postsináptica es menos sensible al efecto de la Aco. Mittag y col., utilizando una técnica de búngarotoxina marcada con $\mathrm{I}^{125}$, demostraron una reducción del 70 al 90\% del número de receptores de Aco en cada unión neuromuscular (2).

La causa del proceso autoinmune se desconoce. Se ha postulado que se inicia por la sensibilización de linfocitos tímicos a los receptores de Aco presentes en las células mioides del timo. Se encuentra hiperplasia del timo entre el 65 y el $75 \%$ de las pacientes y timoma entre el $10 \mathrm{y}$ $15 \%,(1-3)$ benigno en la mayoría de las veces.

Aunque se ha demostrado anticuerpos circulantes en el 85 al $90 \%$ de los adultos con M.G., (3-4) las formas congénitas no tienen anticuerpos circulantes y se consideran de tipo no inmune.

El músculo esquelético suele ser normal, aunque en ocasiones existen agrupaciones perivasculares de linfocitos (linforragias) y excepcionalmente degeneración muscular con intensa respuesta inflamatoria. En estudios ultraestructurales, en la placa motora, se observan alteraciones en los pliegues y en las hendiduras sinápticas. Usando búngarotoxina marcada se comprueba pérdida de receptores de Aco y con técnicas inmunohistoquímicas, se demuestra la existencia de IgG y complemento en la membrana postsináptica. En el 50\% de los casos mortales, se encuentran grupos de fibras esqueléticas degeneradas y parcialmente reemplazadas por tejido fibroso y grasa y ocasionalmente se encuentra necrosis miocárdica focal.

Ahora, la frecuencia afección del timo, los acúmulos linfocitarios perivasculares detectables en tejido muscular, la coincidencia con otras enfermedades autoinmunes 
y la presencia en el suero de estas enfermas de anticuerpos antinucleares (20-40\%) y anticuerpos antitiroideos (30\%), apoyan la patología inmunológica de la M.G. (1).

En la Tabla 1 se puede observar como la M.G. se asocia con múltiples enfermedades, la mayoría de las cuales son autoinmunes (2).

\section{Tabla 1}

\section{ENFERMEDADES VINCULADAS CON M.G.}

\begin{tabular}{|l|l|}
\hline Artritis reumatoide & Dermatitis herpetiforme \\
L.E.S. & Esclerodermia \\
Tiroiditis de Hashimoto & Epilepsia \\
Anemia hemolítica autoinmune & Enfermedad celíaca \\
Polimiositis & Nefritis por c. inmunes \\
Sarcoidosis & Timoma \\
Colitis ulcerosa & Anemia perniciosa \\
S. de Sjögren & Algunas formas de \\
Preeclampsia & psicosis \\
Pénfigo & \\
\hline
\end{tabular}

\section{Clasificación}

La clasificación de las alteraciones miasténicas fue estandarizada por Osserman y col. en 1961 y ampliada al año siguiente por Genkins y col. (ver tabla 2).

\section{Clínica}

La manifestación predominante de la M.G. es la debilidad muscular y la fatigabilidad con el ejercicio, que se recupera parcial o completamente con el reposo o con la administración de drogas A-C.

El inicio puede ser insidioso, pero con frecuencia es subagudo y rara vez agudo. Los músculos de inervación craneal son los más afectados y las manifestaciones clínicas se localizan en el $90 \%$ de los casos a nivel de la musculatura extrínseca del ojo, con ptosis palpebral (que al principio es unilateral), diplopía y oftalmoplejía; las pupilas nunca se afectan. Los músculos faciales, faríngeos y laríngeos se afectan en el $70 \%$ produciendo voz nasal, disartria, disfagia, dificultad respiratoria y una sonrisa conocida como el "gruñido miasténico", debida a la elevación de los labios sin retracción. La invasión de los músculos maseteros puede trastornar la masticación e impedir el cierre de la boca ("signo de la mandíbula colgante"). También se pueden afectar los músculos tríceps, cuadríceps y la musculatura de la lengua y con menor frecuencia la cintura escapular y pélvica y las musculatura de las extremidades con predominio proximal y simétrico $(1,4)$.

La M.G. del recién nacido y de la infancia tiene dos formas: la M.G.N. (neonatal) debida a la transferencia pasiva de anticuerpos de la madre al hijo. La forma juvenil crónica de M.G. puede presentarse en cualquier momento desde el nacimiento hasta la pubertad y se distingue de la M.G.N. por la producción propia de anticuerpos contra receptores de Aco.

Las formas de M.G. del adulto se clasifican de acuerdo con la gravedad de la enfermedad y de la afección o no de los músculos respiratorios.
Tabla 2

CLASIFICACION DE LA M.G.

$\begin{array}{ll}\text { Miastenia neonatal } \\ \text { Miastenia juvenil } \\ \text { Miastenia del adulto: } \\ \text { Etapa I: } & \begin{array}{l}\text { Miastenia ocular pura } \\ \text { Etapa IA: }\end{array} \\ & \begin{array}{l}\text { Síntomas oculares con cambios } \\ \text { electrofisiológicos de generalización }\end{array} \\ \text { Etapa IIA: } & \begin{array}{l}\text { M. generalizada leve, predominantemente } \\ \text { esquelética (brazos y piernas) }\end{array} \\ \text { Etapa IIB: } & \begin{array}{l}\text { M. Generalizada leve con síntomas } \\ \text { bulbares (disfagia, disartria y } \\ \text { dificultad respiratoria). }\end{array} \\ \text { Etapa III: } & \begin{array}{l}\text { M. aguda fulminante, con progreso de los } \\ \text { síntomas oculares hasta incapacidad }\end{array} \\ \text { grave en seis meses. } \\ \text { Etapa IV: } & \begin{array}{l}\text { M. generalizada intensa tardía } \\ \text { Alivio de los síntomas. No hay mejoría } \\ \text { adicional con medicamentos A-C. En la M.G. } \\ \text { Remisión: }\end{array} \\ & \begin{array}{l}\text { de larga duración se pueden presentar } \\ \text { atrofias musculares y cambios axonales, lo } \\ \text { que lleva a déficit funcional (2). }\end{array} \\ & \end{array}$

Las pacientes con M.G. no tienen compromiso sensitivo; el examen neurológico es normal, excepto por la debilidad y la fatigabilidad muscular, con reflejos osteotendinosos normales. La enfermedad toma un curso de remisiones y exacerbaciones espontáneas impredecibles (5).

\section{Diagnóstico:}

1. Clínico: el diagnóstico de la M.G. suele ser obvio tras la historia clínica y la exploración física. Puede detectarse debilidad muscular esquelética en la paciente apretando su mano o flexionando su brazo contra resistencia. La M.G. se confirma con la recuperación parcial o completa de la fuerza muscular luego de unos minutos de reposo y/o por la realización de pruebas con fármacos A-C, así:

\subsection{Cloruro de Edrofonio (Tensilón):}

Esta prueba se basa en el efecto antimiasténico rápido pero breve del cloruro de edrofonio. Se administran dos mg IV y en caso de que no exista reacción de hipersensibilidad, se aplican otros $8 \mathrm{mg}$ más en los 30 segundos siguientes. Se produce franca mejoría de la debilidad muscular en medio a un minuto, volviendo a las condiciones basales en cuatro a cinco minutos. Esta prueba tiene una aplicación especial en las pacientes con afección craneal: los párpados caídos de la oftalmoplejía se recuperan y desaparece la disartria.

\subsection{Prueba de la neostigmina (Prostigmina):}

Se usa cuando no hay disponibilidad del tensilón, cuando falla la prueba con éste o cuando la sintomatología es más acusada en los músculos de las extremidades. Esta droga da una respuesta más lenta, pero su positividad proporciona la evidencia más clara de M.G. Consiste en la inyección IM de 1,5-2 $\mathrm{mg}$ de metilsulfato de 
neostigmina, precedida por una inyección IV de 0,4-0,6 $\mathrm{mg}$ de atropina. La respuesta positiva se aprecia a los $10-$ 15 minutos, pero es máxima a los 30 minutos. También se puede administrar la droga por vía IV o por vía oral (15 mg de bromuro de Neostigmina) (1).

2. Estudios de confirmación complicados incluyen entre otros: ergografía bulbar, electromiografía de fibra única, estudios de conducción nerviosa, estimulación nerviosa repetitiva y valoración de la capacidad vital. Estos estudios determinan el grado de pérdida de fuerza y establecen una línea basal a partir de la cual podemos valorar la respuesta al tratamiento (2).

3. La determinación de anticuerpos antirreceptores de Aco constituyen un criterio diagnóstico importante, aunque tiene algunas limitaciones. Con el empleo de antígenos humanos, un $85-90 \%$ de las enfermas presenta positividad para dichos anticuerpos. No debemos olvidar que la no detección de anticuerpos, no descarta el diagnóstico de M.G. (6). La relación entre clínica y Ac es válida a nivel individual, de tal forma que un paciente en remisión clínica que ha negativizado los anticuerpos es muy poco probable que presente recaída de la enfermedad; por otro lado, una paciente en remisión clínica con anticuerpos positivos es probable que la presente. Ahora, la mayoría de las pacientes seronegativas o con títulos muy bajos corresponden a formas oculares puras; igualmente, las pacientes seronegativas no difieren clínicamente de las seropositivas $(1,7-8)$.

Nuevos recursos tecnológicos permiten determinar anticuerpos contra diferentes componentes de la unión neuromuscular, así:

3.1. Anticuerpos precipitantes: primeros identificados, constituyendo los marcadores más usados: el $80 \%$ de las personas afectadas por M.G., tiene positividad de ellos.

3.2. Anticuerpos bloqueadores de alfa - búngarotoxina: existen dos grupos: los bloqueadores directos y los que dependen de decametonio.

El 98\% de las pacientes con M.G. del adulto, presentan positividad para anticuerpos bloqueadores dependientes de decametonio.

3.3. Anticuerpos anti-idiotípicos: se encuentran en mayor concentración y prevalencia en la M.G. de inicio reciente y se detectan en el $96 \%$ de las pacientes con síntomas de un año o menos. Sólo el $50 \%$ de pacientes con M.G. de más de cinco años, tienen este anticuerpo (2).

Además de los anticuerpos contra receptores de Aco, se pueden observar otras alteraciones serológicas como: anticuerpos anti-músculo estriado, presentes sobre todo en pacientes con M.G. más timoma.

Dado el contex to inmunológico de la enfermedad y su posible asociación a otras enfermedades autoinmunes, es bueno determinar pruebas de función tiroidea, anticuerpos antitiroideos, anticuerpos antinucleares (ANA) y presencia de factor reumatoide.

Por último, debe buscarse siempre la existencia de timoma. La radiografía clásica se ha sustituido por la TC torácica, técnica de elección con la que no se presentan falsos negativos (1).

\section{M.G. y embarazo:}

Dada la tendencia de la enfermedad a presentarse durante los años reproductivos de la vida, la asociación entre embarazo y M.G. reviste especial importancia para el obstetra. Hace varias décadas una mujer embarazada con M.G. tenía un riesgo significativo de desarrollar complicaciones que amenazarán su vida. Aún hoy en día, aunque el manejo ha mejorado dramáticamente, el tratamiento puede provocar efectos adversos en la gestante y su recién nacido.

\section{Efectos del embarazo sobre la M.G.:}

E1 embarazo tiene un efecto impredecible sobre la evolución de la M.G., con porcentajes iguales de pacientes que mejoran, empeoran o no sufren modificaciones $(5,9)$. La evolución puede ser diferente durante distintos embarazos en una misma mujer. Warren Plauché y col. (2) revisan 322 embarazos en 225 miasténicas publicados en la literatura mundial, encontrando (ver tabla 3):

Tabla 3

EFECTOS MATERNOS EN 322 EMBARAZOS DE 225 MADRES CON M.G.

\begin{tabular}{|lrr|}
\hline & No. & $\%$ \\
\hline Exacerbaciones durante el embarazo & 132 & 41,0 \\
Remisiones durante el embarazo & 92 & 28,6 \\
Sin cambios durante el embarazo & 102 & 31,7 \\
Exacerbaciones postparto & 96 & 29,8 \\
Muertes maternas & 9 & 4,0 \\
\hline
\end{tabular}

Como no hay un patrón definido y dada la propensión de las embarazadas a presentar exacerbaciones súbitas y devastadoras, con frecuencia acompañadas de insuficiencia respiratoria, sobre todo en el postparto, los obstetras deben estar atentos ante la posibilidad de empeoramiento de la gestante en cualquier momento, monitorizándola estrictamente durante el embarazo, trabajo de parto, parto y puerperio $(2,9)$.

\section{Cambios fisiológicos que afectan la M.G.:}

Muchos cambios del embarazo pueden afectar la evolución de la M.G., así: las náuseas y vómito de las etapas iniciales, el temor a tomar drogas durante la gestación, el aumento de la depuración renal y del volumen sanguíneo y la absorción errática de los medicamentos, pueden alterar las dosis necesarias de fármacos A-C para controlar la M.G.

Por otra parte, la ansiedad y el estrés fisiológico vinculados al embarazo, pueden ser responsables en parte de las exacerbaciones de la M.G.

Igualmente, los segmentos basales pulmonares no se distienden por completo en la mayoría de las gestantes, debido a la elevación del diafragma por el útero en crecimiento; la disminución del movimiento diafragmático y la hipoventilación resultante constituyen un riesgo real en miasténicas, cuyos músculos respiratorios son debilitados por la enfermedad. 
Brenner citado por Plauché (2) identifica a la alfa fetoproteína (AFP) como inhibidora de los anticuerpos contra los receptores de la Aco. Ahora, si las titulaciones crecientes de AFP protegen a la madre de manifestaciones de M.G., sería de esperar el menor número de síntomas y casi todas las remisiones en etapas tardías del embarazo, cuando las titulaciones de AFP suelen ser máximas. Las exacerbaciones tendrían más posibilidad de presentarse, como de hecho lo hacen, después del parto, cuando las cifras de AFP disminuyen de manera precipitada (10). Las infecciones juegan un papel fundamental en la aparición de las exacerbaciones graves de la M.G., por tanto se deben tratar pronto y adecuadamente las infecciones respiratorias y las infecciones urinarias, incluyendo a la bacteriuria asintomática; igualmente incrementar la vigilancia en las pacientes que reciben esteroides, dada su susceptibilidad a las infecciones.

\section{Trabajo de parto:}

El estrés físico del trabajo de parto y parto aumentan la debilidad miasténica. Es necesario observar cuidadosamente a la paciente en trabajo de parto en busca de signos de alteración respiratoria e hipooxigenación. El ejercicio debe disminuirse al mínimo y se debe disponer de sostén respiratorio inmediato. Durante el trabajo de parto deben administrarse los fármacos A-C por vía parenteral, dada su absorción digestiva impredecible por vía oral y el vaciamiento gástrico prolongado.

Deben evitarse todas las drogas con efectos similares al curare o aquellas que agraven o precipiten los síntomas y signos de la M.G. Ver tabla 5.

En caso de hipodinamia hay buena respuesta a los oxitócicos. El dolor se puede aliviar con Demerol. Si se desarrolla hipertensión inducida por el embarazo, la administración de sulfato de magnesio está contraindicada, ya que puede bloquear la liberación de Aco en la unión neuromuscular. La duración del trabajo de parto de las pacientes miasténicas no difiere de la de las pacientes sin enfermedad, $(2,5)$ aunque varios autores comunican una menor duración y relativa ausencia de dolor en el trabajo de parto en casos individuales (9).

Es importante la vigilancia fetal en cuanto a signos de hipoxia durante cualquier exacerbación o crisis de la M.G.

Debemos recordar que el músculo liso uterino no participa del proceso miasténico, ya que su mecanismo contráctil es independiente de receptores de Aco. Durante el expulsivo, por el contrario, cuando los músculos estriados de la pared abdominal, responsables del pujo, son usados, los efectos de la M.G. pueden hacerse más notables; el obstetra debe estar preparado para ayudar a la paciente cansada, con la aplicación de un fórceps bajo o terminal.

La operación cesárea sólo está justificada por razones obstétricas: recordar que las intervenciones quirúrgicas en M.G. implican estrés, interrupción del medicamento y programas de reposo, así como los peligros de los agentes relajantes y los anestésicos. Igualmente, el postoperatorio causa movilidad diafragmática disminuida por el dolor a nivel de la incisión y un difícil control de las secreciones bronquiales, por lo que podemos concluir que el parto quirúrgico es particularmente peligroso para la madre miasténica $(2,9)$.

\section{Efectos de la M.G. sobre el embarazo:}

Las tablas 3 y 4 muestran como tanto la madre miasténica como su hijo tienen riesgos mucho mayores durante el embarazo. La madre puede presentar: insuficiencia respiratoria, respuestas adversas a los fármacos, exacerbación de la M.G., crisis miasténica e incluso la muerte. La incidencia de toxemia en combinación con M.G. nø es más alta que en la población general (9). El feto presenta prematurez y miastenia gravis neonatal: el riesgo de prematurez y/o de trabajo de parto prematuro puede aumentar ligeramente en las mujeres con M.G.; los reportes de prematuridad van del 13 al 41,3\%, mientras que en la población general están entre 8,8 y $24,8 \%$ (9). Reportes de malformaciones congénitas en niños de madres con M.G., quienes no recibieron esteroides u otros inmunosupresores son raros e incluyen: artrogriposis múltiple congénita, hipognatismo, polidactilia e hipogama-globulinemia. Estas anomalías son tan infrecuentes que no pueden atribuirse a la M.G. sola (9).

\section{Relaciones con el aborto: ver tabla 4 .}

La terminación del embarazo tiene muy poca utilidad en el tratamiento de la miasténica embarazada. Hay, citado por Plauché (2) considera que con el aborto terapéutico en pacientes con exacerbaciones de la M.G. en el primer trimestre, no podría predecirse un beneficio permanente de la M.G. y que por el contrario podría ocurrir una exacerbación por el procedimiento anestésico, la cirugía o durante la recuperación.

Tabla 4

EFECTO SOBRE FETOS Y RECIEN NACIDOS EN 322 EMBARAZOS DE 225 MADRES CON M.G.

\begin{tabular}{|lrc|}
\hline & No. & $\%$ \\
\hline Aborto & 38 & 11,8 \\
$\quad$ Espontáneo & $(14)$ & \\
$\quad$ Inducido & $(24)$ & \\
Nacidos Vivos & 276 & 85,6 \\
Muertes Perinatales & 22 & 6,8 \\
Obitos & $(8)$ & \\
Muertes Neonatales & $(14)$ & \\
Miastenia Neonatal & 48 & 14,9 \\
\hline
\end{tabular}

\section{Analgesia y anestesia:}

La paciente miasténica es particularmente sensible a sedantes, narcóticos y tranquilizantes, los cuales se pueden administrar con seguridad en dosis ajustadas a la necesidad y respuesta. Debe evitarse la depresión respiratoria y la aspiración de secreciones bronquiales.

Rolbin y col. en una revisión de los problemas anestésicos en las gestantes con M.G., recalcan la importancia de la valoración preanestésica, la cual incluye: un EKG (dada la presentación de necrosis miocárdica focal 
en algunas pacientes), estudios de función pulmonar (para identificar cualquier disfunción respiratoria y ayudar en el tratamiento de la insuficiencia cuando se presente) y pruebas de función tiroidea (en las pacientes a quienes se les sospecha coexistencia de enfermedad tiroidea) (11).

Igualmente recomiendan la anestesia epidural para disminuir los requerimientos de medicamentos sistémicos, evitar la fatiga, controlar la ansiedad y proporcionar buena anestesia para la aplicación del fórceps bajo con el propósito de abreviar el expulsivo.

En gestantes miasténicas con afección bulbar o respiratoria que vayan a ser sometidas a cesárea, recomiendan anestesia endotraqueal general, ya que es más fácil controlar las vías aéreas, la oxigenación y las secreciones; igualmente evita la complicación de bloqueos motores altos.

Las miasténicas son muy sensibles a los relajantes musculares no despolarizantes: el curare y la succinilcolina son motivo de controversia en el tratamiento anestésico de pacientes con M.G.

En la tabla 5 se incluyen algunos anestésicos y drogas que precipitan o aumentan los síntomas y signos de la M.G. y que por lo tanto están contraindicados $(1-2,9)$.

Tabla 5

FARMACOS QUE AFECTAN A LA M.G.

\begin{tabular}{|ll|}
\hline Antibióticos & Anticonvulsivantes \\
Neomicina & Fenitoína \\
$\begin{array}{l}\text { Aminoglicósidos } \\
\text { Kanamicina } \\
\text { Gentamicina } \\
\quad \text { Estreptomicina }\end{array}$ & Trimetadiona \\
$\quad$ Tobramicina & Drogas Sicotrópicas \\
Polimixina B & \\
Colisticina & Sales de Litio \\
Oxitetraciclina & Clorpromazina \\
Lincomicina & \\
Quindamicina & Hormonas \\
(Ciprofloxacina) & \\
Drogas cardiovasculares & ACTH \\
Lidocaína & H. Tiroidea \\
Quinina & Anestésicos Inhalatorios \\
Quinidina & Eter \\
Procainamida & Cloroformo \\
Propranolol & Fluotane \\
Oxiprenolol & Tricloroetileno \\
Practolol & Otras Drogas \\
Drogas Antirreumáticas & Barcóticos \\
D-Penicilamina & Barbitúricos \\
Cloroquina & Tranquilizantes \\
& \\
\hline
\end{tabular}

El tratamiento postanestésico es crítico para las embarazadas miasténicas. Son frecuentes las exacerbaciones en el postparto, por lo que se requiere valorar la capaci- dad ventilatoria y los gases arteriales con frecuencia y eliminar las secreciones bronquiales. Los primeros días postoperatorios deben ser vigilados idealmente en una unidad de cuidados intensivos.

El Sulfato de Magnesio utilizado en obstetricia para prevención de convulsiones eclámpticas y tocólisis, disminuye la acción despolarizante de la Aco, disminuye las sustancias transmisoras en la placa terminal motora y deprime la excitabilidad de la membrana muscular; por tanto está contraindicado en las embarazadas miasténicas, ya que puede precipitar las crisis.

La miocardiopatía latente en algunas pacientes miasténicas aumenta el riesgo de uso de agentes betamiméticos como la ritodrina y la terbutalina; de ahí la necesidad de practicar E.K.G. a las pacientes que se van a someter a terapia úteroinhibidora.

\section{Modalidades terapéuticas en la M.G.:}

Se deben considerar dos aspectos terapéuticos diferentes: uno se refiere a los fármacos utilizados para tratar los síntomas de la paciente, pero que no actúan sobre la enfermedad en sí (anticolinesterásicos), y el otro a la utilización de tratamiento específico para la enfermedad (timectomía, corticoides, inmunosupresores). La plasmaféresis se considera como una medida terapéutica intermedia (1).

\section{Drogas anticolinesterasa:}

Los fármacos A-C (compuestos de amonio cuaternario) deben emplearse siempre en primera instancia, dada su gran efectividad y escasa toxicidad; desgraciadamente sólo consiguen remisiones en forma poco graves de M.G. y en las formas oculares. Los más empleados son la Neostigmina (prostigmina) y la piridostigmina (mestinón). Debe emplearse uno u otro, pero no deben asociarse, ya que no se consiguen mejores resultados.

Recordar que los fármacos $\mathrm{A}-\mathrm{C}$ a menudo no pueden restablecer la fuerza óptima cuando la M.G. es intensa; de ahí que sea necesario advertir a las pacientes que el incremento de la dosis no aumentará la fuerza. Las dosis excesivas de fármacos $\mathrm{A}-\mathrm{C}$ producen efectos muscarínicos desagradables como: cólicos abdominales, flatulencia, diarrea, náuseas, vómito, salivación y lagrimeo; efectos avanzados de sobredosis son: debilidad muscular paradójica e insuficiencia respiratoria, que pueden ser mortales.

El primer fármaco A-C utilizado en el tratamiento de 1a M.G. fue la neostigmina (prostigmina). La dosis es de $15 \mathrm{mg}$ cada dos - tres horas, ajustándolo con frecuencia a aquella cifra con la que la fuerza muscular sea óptima, con un mínimo de efectos secundarios colinérgicos. Se valora la capacidad vital o la fuerza prensil del puño para determinar los efectos farmacológicos. La principal limitación terapéutica de la neostigminia es su breve duración de acción (dos-tres horas). Muchas pacientes responden mejor a preparados de larga duración de acción: el más usado es la piridostigmina (mestinón), $60 \mathrm{mg}$ cada cuatro-seis horas. La forma de liberación sostenida de piridostigmina llamada timespan, permite a las pacientes dormir toda la noche sin despertar para tomar 
medicamentos; igualmente es posible la actividad social durante varias horas sin aparición de ptosis palpebral, disartria o pérdida de la fuerza muscular de las piernas. En la tabla 6, se muestra la dosis equivalentes de fármacos A-C de uso frecuente. $(2,12)$.

\section{Tabla 6}

\section{DOSIS EQUIVALENTES DE A-C}

\author{
$0,5 \mathrm{mg}$ de Neostigmina IV \\ 1,5 mg de Neostigmina Subcutánea \\ $15,0 \mathrm{mg}$ de Neostigmina V.O. \\ $60,0 \mathrm{mg}$ de Piridostigmina V.O. \\ 180,0 mg de Mestinón Timespán V.O. (una vez/día)
}

Si se utiliza la vía IM, la dosis total debe ser la décima parte de la que recibía por vía oral, y si la administración es IV, debe reducirse 30 veces.

Todas las embarazadas miasténicas se controlan perfectamente con drogas A-C, requiriendo ajustes frecuentes de la dosis, ya que los cambios fisiológicos que ocurren durante el embarazo, vistos anteriormente, modifican las cifras sanguíneas y la duración de la actividad del fármaco. Si no se consiguen los efectos terapéuticos deseados, se debe aumentar la dosis, reduciendo primero el intervalo entre éstas; si esto no es suficiente, se incrementa la dosis de cada toma: 5 a $10 \mathrm{mg}$ de neostigmina o 15-30 mg de piridostigmina. No olvidar que la sintomatología clínica excepcionalmente remite en forma total, por lo que las modificaciones en la dosis sólo se harán hasta conseguir un claro efecto beneficioso (2).

$\mathrm{Si}$ la evolución del paciente con drogas A-C y timectomía no es correcta o tampoco lo es en aquellas pacientes mayores de 40 años sin timectomía y con solo tratamiento a base de A-C, podemos agregar esteroides. Se debe tener precaución con el uso de fármacos A-C por vía parenteral, ya que son más frecuentes la sobredosis y las crisis colinérgicas, reservándose esta vía parenteral para el manejo de las dosis durante el trabajo de parto, por los motivos ya expuestos.

\section{Corticosteroides:}

El mecanismo de acción de la prednisona en la M.G. es desconocido, aunque se supone que está relacionado con su efecto inmunosupresor. Las pacientes miasténicas que no responden al tratamiento con fármacos $\mathrm{A}-\mathrm{C}$, pueden beneficiarse de los esteroides. Son además particularmente útiles en adolescentes y adultos jóvenes y en todas las formas autoinmunes de M.G. asociadas o no a timomas. Se debe iniciar con dosis bajas ( $25 \mathrm{mg}$ ), los cuales se van aumentando progresivamente con el fin de evitar la agravación del bloqueo neuromuscular, hasta llegar a $60-80 \mathrm{mg} / \mathrm{día}$ (un $\mathrm{mg} / \mathrm{kg}$ ). Posteriormente y según la evolución, la dosis se disminuye progresivamente, hasta pasar a dosis alternas. Otros autores prefieren utilizar las dosis alternas desde el inicio del tratamiento, con el objeto de evitar los efectos inhibidores de los esteroides sobre el funcionamiento hipotálamohipofisiario; en estos casos se inicia con $100 \mathrm{mg}$ de prednisona en días alternos, para ir reduciendo gradual- mente a medida que mejora la enfermedad (cinco $\mathrm{mg} / \mathrm{día}$ cada cuatro-seis semanas) hasta la dosis mínima de mantenimiento (1-2).

Recordar que la 11 deshidrogenasa placentaria inactiva el $50 \%$ de la prednisona, el $70 \%$ de la cortisona e hidrocortisona y que la beta y la dexametasona casi no se modifican y cruzan libremente la placenta (13).

Desafortunadamente pocas miasténicas pueden mantener su remisión al interrumpir los esteroides, por lo que se recomienda continuar con ellos para evitar recidivas; de ahí que las pacientes que se embaracen con este tratamiento, deben mantenerlo a la dosis más bajas que controlen la enfermedad y eviten las exacerbaciones. Los esquemas de administración cada tercer día son eficaces por disminuir al mínimo la dosis de esteroides. Los riesgos del labio leporino y paladar hendido son mínimos.

Sanders citado por Plauché (2) insiste en que uno de los momentos más seguros para que las miasténicas se embaracen es el de la remisión por esteroides; igualmente agrega que el parto y los procedimientos quirúrgicos son más fáciles de atender en la miasténica que logró un estado de remisión con la administración de los mismos. No hubo exacerbaciones postparto en las tres madres miasténicas de Sanders, cuyos embarazos tuvieron respuestas estables a esteroides.

\section{ACTH:}

Se ha recomendado ACTH para el control de las crisis miasténicas; al principio del tratamiento suele empeorar la debilidad, efecto transitorio seguido de mejoría prolongada en algunos casos.

\section{Antimetabolitos:}

Se pueden usar otros agentes inmunosupresores cuando no hay respuesta a los esteroides. Los fármacos antimetabolitos son inmunosupresores comprobados en miasténicas no embarazadas. La azotioprina (inmuran) es el más utilizado, pero en raras ocasiones es de primera línea. Casi el 33\% de las pacientes mejoran con inmunosupresores y un número equivalente tiene efectos secundarios considerables.

\section{Gammaglobulinas:}

Genkins y col. comunicaron 57 casos de M.G. en las que se administró 10 c.c. semanales de gammaglobulina como coadyuvante, sin efectos adversos. En la miastenia ocular la GG combinada con esteroides permitió disminuir la dosis de estos últimos.

\section{Timectomía:}

La timectomía está indicada:

- En todos los casos de timona y

- En las formas generalizadas de M.G.

- Así mismo, y dada la asociación de M.G. con timitis en pacientes menores de 40 años, la timectomía es la segunda medida a seguir antes de utilizar esteroides o inmunosupresores.

La mejoría clínica se presenta en el $75-85 \%$ de las pacientes, aún en aquellas sin timoma, pero la respuesta 
es tardía (aún después de varios años). Los enfermos con timoma evolucionan peor, sea o no extirpado el tumor.

Definitivamente no está indicada en las formas oculares puras, en adolescentes o en ancianos.

Cuando se practica la timectomía, se aconseja mantener la dosis de fármacos $\mathrm{A}-\mathrm{C}$ hasta el día fijado para la cirugía, fecha en la cual se suspende toda medicación. Si el paciente recibía tratamiento con prednisona antes de la intervención, deben administrarse dosis equivalentes por vía parenteral. Luego de la intervención quirúrgica, debe reiniciarse el tratamiento con fármacos $\mathrm{A}-\mathrm{C}$ a mitad de la dosis preoperatoria como única medicación, reajustando la dosis, de acuerdo con la evolución de la paciente (1-3).

Ip y col. citados por Plauché (2) comunicaron en 1986 el primer caso de timectomía para tratamiento de la crisis miasténica durante el embarazo: a esta paciente se le practicó timectomía transesternal a las 17 semanas de gestación; su estado general se estabilizó, aunque necesitó piridostigmina en dosis elevadas durante el postoperatorio. Su embarazo concluyó en un parto normal a las 39 semanas.

\section{Plasmaféresis:}

Indicada de preferencia en las crisis miasténicas y como terapéutica previa a la timectomía, permiten lograr remisiones de corta duración relacionadas con la disminución de los anticuerpos circulantes contra receptores de Aco.

Dau citado por Planché (2) informó de 35 pacientes con M.G. tratadas con una combinación de plasmaféresis repetida, esteroides y azotioprina; su esquema incluía intercambios de plasma de dos-tres litros, dos a siete veces por semana hasta observar mejoría. Este y otros autores concluyen que la plasmaféresis más la terapia inmunosupresora constituye una poderosa modalidad terapéutica para crisis de M.G.

Menke y col. proporcionan guías excelentes para el cuidado de las pacientes sometidas a plasmaféresis; los problemas más frecuentes fueron: mayor susceptibilidad a las infecciones, sobre todo neumonía por gram negativos e infecciones virales diseminadas (2).

Levine y col. comunican en 1986 la primera utilización exitosa de la plasmaféresis en una M.G. fulminante durante la gestación (14). La plasmaféresis tendría importancia particular, si los títulos de anticuerpos maternos elevados predijeran con precisión la aparición de M.G. neonatal.

\section{Crisis miasténica y tratamiento:}

Las pacientes con M.G. pueden presentar tres tipos de crisis:

1. Crisis miasténica: es rara en la paciente bien tratada. Se define como crisis miasténica a una exacerbación de los síntomas que requiere asistencia ventilatoria mecánica. Suele ser precipitada por el estrés de una intervención quirúrgica, enfermedad, infecciones, el parto, el ejercicio físico intenso, las emociones fuertes, los fármacos ya mencionados (Tabla 5) y los cambios en los medicamentos A-C.
En caso de presentarse una crisis miasténica, se debe administrar neostigmina por vía IM y en los casos de difícil control, practicar plasmaféresis. Debe mantenerse la función respiratoria con intubación endotraqueal y ventilación asistida.

2. Crisis colinérgica: debida a una sobredosis de fármacos A-C. Estas pacientes presentan síntomas digestivos muscarínicos intensos, debilidad e insuficiencia respiratoria. El índice más fiable es el diámetro pupilar, el cual no debe ser menor de dos $\mathrm{mm}$ con luz ambiental. En casos graves aparece bradicardia, hipotensión, confusión y coma. Para evitar estos efectos se aconseja la administración conjunta de 0,4 a $0,6 \mathrm{mg}$ de atropina IV.

3. Crisis reactiva: aquella en la que la paciente se vuelve refractaria a fármacos $\mathrm{A}-\mathrm{C}$.

\section{Miastenia Gravis Neonatal (M.G.N.):}

Strickroot y col. comunicaron por primera vez M.G.N. en 1942. Del 10 al $20 \%$ de los recién nacidos de madres con M.G. presentan M.G.N. Los síntomas aparecen 12 a 48 horas después del nacimiento, pero pueden presentarse tan tarde como en el tercer día: éstos incluyen: succión y llanto débiles, disminución en los movimientos espontáneos y sufrimiento respiratorio; en casos excepcionales presentan parálisis respiratoria y mueren. Los que sobreviven tendrán una enfermedad autolimitada a 1-12-15 semanas $\sin$ ulteriores recaídas.

El retraso en el inicio de la M.G.N. se puede relacionar con la transferencia de fármacos $\mathrm{A}-\mathrm{C}$ hidrosolubles a partir de la madre o con una concentración inicial alta de AFP rápidamente decreciente $(1,9)$.

La M.G.N. es el resultado de la transferencia pasiva de anticuerpos maternos contra receptores de Aco al feto, cuya concentración disminuye gradualmente al tiempo que remite su enfermedad; las madres cuyos títulos de anticuerpos son muy altos, tienen un mayor riesgo de hijos afectados. No debe considerarse a estas titulaciones como marcadores absolutos de M.G.N., sin embargo, se debe tener en cuenta la disminución de anticuerpos maternos muy aumentados por plasmaféresis, con el fin de disminuir el riesgo de M.G.N. Recordar que las cifras de anticuerpos en el recién nacido no tienen relación constante con los de la madre; es así como se ha descrito M.G.N. en el hijo de una miasténica en remisión, siendo los títulos de anticuerpos de esta señora tan altos como en la miastenia activa.

Como ya se mencionó, la AFP inhibe los anticuerpos contra R Aco. Las concentraciones protectoras del recién nacido de una madre miasténica disminuyen después del parto, de ahí la rareza de signos de miastenia en el feto in útero y en el período latente que precede a la M.G.N. (2).

Toda la sintomatología del recién nacido responde rápidamente a la administración parenteral de fármacos A-C, aunque lo más adecuado es conseguir una adecuada nutrición. Es necesario observar cuidadosamente a los recién nacidos de madres con M.G. buscando signos de debilidad muscular esquelética, en particular de los músculos respiratorios y de la deglución; el recién nacido pudiera requerir apoyo con fármacos $\mathrm{A}-\mathrm{C}$ hasta que desaparezca tal debilidad, por lo general en tres semanas. 
La insuficiencia respiratoria súbita constituye un riesgo importante en los neonatos afectados. Se ha sugerido la plasmaféresis temprana para el tratamiento del raro recién nacido muy afectado.

\section{Miastenia congénita:}

Implica el diagnóstico de miastenia casi siempre basada en una oftalmoplejía bilateral en un recién nacido de madre sin M.G. Al contrario de lo que ocurre en la M.G.N., los movimientos fetales son muy débiles, no existen remisiones clínicas y se aprecia una tendencia a la mejoría clínica después de los 8-10 años de vida. Se comporta igual a la M.G: del adulto. En muchos casos no se demuestra la presencia de anticuerpos contra RAco. La respuesta a anticolinérgicos es incierta y la timectomía no mejora el cuadro clínico. La evolución natural de la enfermedad es de curso benigno.

\section{Lactancia:}

Los anticuerpos contra RAco de la madre con M.G. pasan al recién nacido en la leche e incrementan potencialmente la M.G.N. Los fármacos A-C presentes en la leche, pueden causar trastornos digestivos en el recién nacido como cólicos; sólo pequeñas cantidades de esteroides e inmunosupresores aparecen en la leche materna.

En general se puede concluir, que una madre miasténica en remisión, con bajas titulaciones de anticuerpos y sin tratamiento peligroso para el recién nacido, puede amamantarlo con seguridad. Los títulos elevados de anticuerpos, dosis altas de A-C o la exacerbación de los síntomas de M.G. impiden la lactancia (2).

\section{Resumen}

La M.G. es una enfermedad autoinmune compleja, que se caracteriza por la producción de anticuerpos ( $\operatorname{IgG})$ que destruyen la RAco de la membrana postsináptica de la unión neuromuscular, lo cual disminuye la transmisión de los impulsos nerviosos en los grupos musculares afectados. Clínicamente se manifiesta por disminución de la fuerza muscular y cansancio rápido con el ejercicio.

El embarazo tiene un efecto impredecible sobre la evolución de la M.G.; sin embargo, cuando se presentan las crisis o las exacerbaciones, lo hacen de una manera súbita y devastadora, sobre todo en el postparto (al parecer relacionado con una disminución brusca de las cifras de AFP).

Las gestantes miasténicas se controlan perfectamente con fármacos $\mathrm{A}-\mathrm{C}$, pero debido a los cambios fisiológicos del embarazo, las dosis requieren ajustes frecuentes. Igualmente, los fármacos A-C y los esteroides son primordiales en el manejo médico de la M.G. materna. La timectomía, la plasmaféresis, los inmunosupresores, la gamaglobulina y la ACTH son coadyuvantes de utilidad diversa. La M.G. produce aumento de la morbimortalidad materna, de las pérdidas gestacionales, del T. de P. prematuro y de la M.G.N.

La mortalidad materna es de aproximadamente $40 \mathrm{x}$ 1.000 y la perinatal de $68 \times 1.000$ nacidos vivos (cinco veces más que la de los embarazos no complicados).

El embarazo no se contraindica en las miasténicas, y una buena evolución depende de la vigilancia estricta materna y fetal (durante el embarazo, parto y puerperio) y de la detección temprana y el manejo adecuado de las exacerbaciones y las crisis miasténicas.

\section{BIBLIOGRAFIA}

1. Urbano Márquez, A. y Grau, J.M. Enfermedades de la unión neuromuscular: Miastenia grave y síndromes miasténicos. En: Farreras Valenti, P. y Rozman, C. Medicina Interna. Barcelona (Espana). Ediciones Doyma. Duodécima edición; 1992; 1523-1527.

2. Plauché WC. Miastenia grave en madres y sus recién nacidos. Clínicas Obstétricas y Ginecológicas, 1991; 1: 79-94.

3. Camacho LM., Vergara I. Enfermedades musculares. Enfermedades de la unión neuromuscular: Miastenia Gravis (M.G.). En: Chalem, F., Escandón JE., Campos J. y Esguerra R. Medicina Interna. Vol. II. Colombia. Impresión Editorial Presencia Ltda., segunda edición, 1992; 1166-1168.

4. Bradley WG., Adams RD. Miastenia grave, trastornos de la unión neuromuscular y debilidad muscular episódica. En: Harrison. Principios de Medicina Interna. Tomo II. México. Libros MC Graw-Hill sexta edición, 1986; 3062-3066.

5. Bellur SN. Patología de los músculos estriados en el embarazo: Miastenia Gravis. En: Gleicher, N. Medicina Clínica en Obstetricia. Tomo II. Buenos Aires (Argentina). Editorial Médica Panamericana, 1989; 1064-1070.

6. Mossman S., Vincent A. y Newson Davis Jr. Myasthenia gravis without acetylcholine receptor antibody: a distinct disease entity. Lancet 1986; 1: 116.
7. Illa I., Rodríguez JL., Grau JM y Juárez C. Anticuerpos antirreceptor de acetilcolina en miastenia grave. Inmunología 1988; 7: 21-26.

8. Soliven BC., Lange DJ., Penn AS. Seronegative myasthenia gravis. Neurology 1988; 38: 514-517.

9. Fennell DF., Ringel SP. Myasthenia gravis and pregnancy. Obstetrical and Gynecological Survey 1987; 41(7): 414-421.

10. Hatada Y., Munemura M., Matsuo I. Myastenic crisis in puerperium: the possible importance of alpha-fetoprotein: case report. Br. J. Obstet. Gynaecol. 1987; 94: 480.

11. Rolbin SH., Levinson G., Shnider SM. Anesthetic consideration for myasthenia gravis and pregnancy. Anesth Analg 1978; 57: 441.

12. Dalessio DJ. Complicaciones neurológicas: miastenia grave en el embarazo. En: Burrow-Ferris. Complicaciones médicas durante el embarazo. Buenos Aires (Argentina). Editorial Médica Panamericana, 1977; 502-504.

13. Blanford AT., Murphy SEP. In vitro metabolism of prednisona, dexamethasone, betamethasone and cortisol by the human placenta. Am. J. Obstet. Gynecol. 1977; 127: 264.

14. Levine SE., Keesey JC. Successful plasmapheresis for fulminant myasthenia gravis during pregnancy. Arch Neurol 1986; $42: 197$. 